\title{
Winning at hide-and-seek with latent HIV reservoirs
}

acitretin, either alone or in combination with SAHA, substantially reduced HIV DNA levels and increased apoptosis in CD4 ${ }^{+}$cells
Latent reservoirs of HIV particles have been a major obstacle to the development of curative therapies for this disease. A paper in Nature Medicine suggests that increasing the expression of retinoic acidinducible gene I protein (RIG-I), a pattern-recognition receptor that induces apoptosis of virus-infected cells, could be used alongside other latency-reversing agents (LRAs) to destroy these reservoirs.

Although antiretroviral therapy (ART) can be used to maintain low viral loads in HIV-infected individuals, HIV particles that are not actively transcribed can lay dormant and re-emerge upon cessation of ART. LRAs, such as the histone deacetylase inhibitor suberoylanilide hydroxamic acid (SAHA), generally aim to increase transcription of latent viral RNA, which should activate pattern recognition receptors and thus stimulate the innate immune system, which then lyses the infected cell.

Although many LRAs indeed increase transcription of latent HIV RNA, they fail to activate innate immunity and induce cell death; the sequestration and degradation of RIG-I in HIV infected individuals could contribute to this failure, as RIG-I activates the innate immune response. Li and colleagues reasoned that increasing RIG-I expression could enable the detection of HIV RNA and the elimination of infected cells. To do this, they used acitretin - an FDA-approved retinoic acid derivative used to treat psoriasis to stimulate RIG-I transcription.

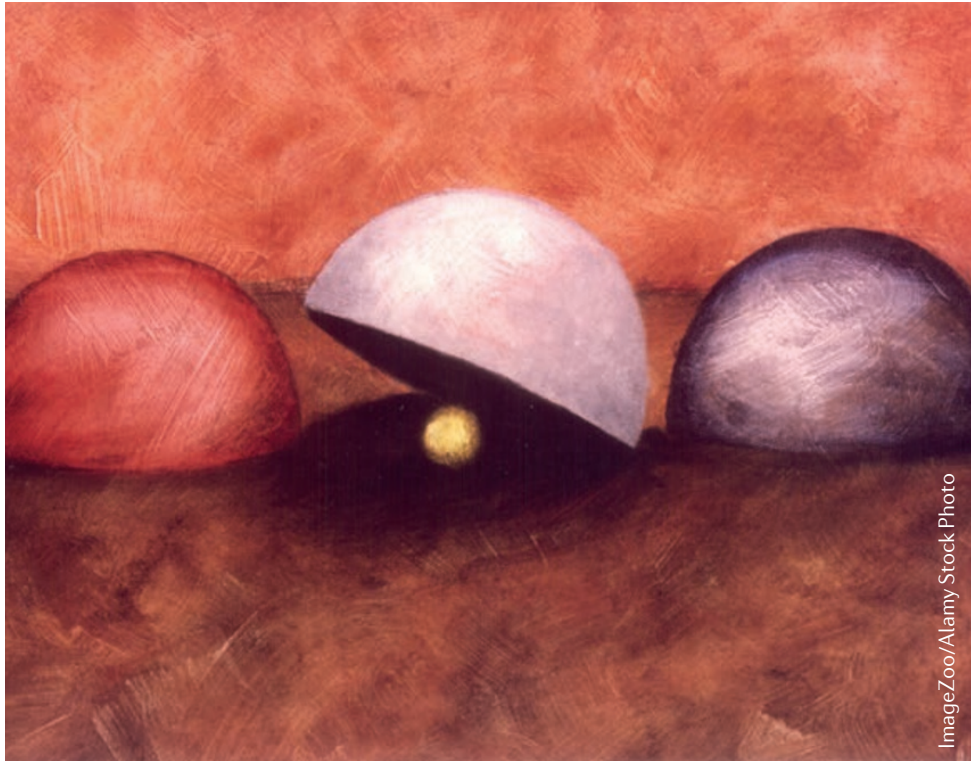

Retinoic acid also activates p 300 acetyl transferase, which could simultaneously increase HIV transcription.

In human primary $\mathrm{CD} 4^{+} \mathrm{T}$ cells induced with HIV, acitretin and SAHA both increased the expression of HIV RNA. Acitretin treatment reduced cellular HIV DNA levels. HIV DNA was undetectable in cells treated with both acitretin and SAHA, suggesting that this combination induces substantial cell death. The observed cell death was probably caused by apoptosis, as shown by annexin $\mathrm{V}$ and caspase 3 staining.

Similarly, acitretin, either alone or in combination with SAHA, substantially reduced HIV DNA levels and increased apoptosis in $\mathrm{CD} 4^{+}$ cells from patients receiving ART, effects that have not been observed with other candidate LRAs.

No increase in apoptosis was observed in healthy T cells.

Acitretin treatment also increased the secretion of interferon- $\beta$ and CXC chemokine ligand 10 (CXCL10) from infected (but not uninfected) cells, further contributing to the death of infected cells.

Taken together, these data indicate that acitretin, particularly in combination with SAHA, could be an effective strategy to destroy HIV reservoirs in infected patients, paving the way to a curative therapy.

Megan Cully

ORIGINAL ARTICLE Li, P. et al. Stimulating the RIG-I pathway to kill cells in the latent HIV reservoir following viral reactivation. Nat. Med. http://dx.doi.org/10.1038/nm.4124 (2016) 